\title{
Criminologie
}

\section{Le dédommagement des victimes}

\section{Alice Parizeau}

Volume 13, numéro 1, 1980

Regards sur la victime

URI : https://id.erudit.org/iderudit/017115ar

DOI : https://doi.org/10.7202/017115ar

Aller au sommaire du numéro

Éditeur(s)

Les Presses de l'Université de Montréal

ISSN

0316-0041 (imprimé)

1492-1367 (numérique)

Découvrir la revue

Citer cet article

Parizeau, A. (1980). Le dédommagement des victimes. Criminologie, 13(1),

37-37. https://doi.org/10.7202/017115ar d'utilisation que vous pouvez consulter en ligne.

https://apropos.erudit.org/fr/usagers/politique-dutilisation/ 
Le principe du dédommagement des victimes n'est pas nouveau en droit. En effet, en Grande-Bretagne avant l'avènement de la common law, un individu qui a lésé un autre, pouvait essayer de compenser le dommage causé, et s'entendre avec la victime, au lieu d'avoir à répondre de ses actes devant une cour de justice. Il est vrai également, qu'il s'agit là d'une pratique qui a toujours existé, dans le milieu rural, par exemple, où il est relativement fréquent que les voisins s'entendent sur un dédommagement à l'amiable en vue de compensation pour les actes de vandalisme, d'incendie, ou de vol, commis par des jeunes, entre autres. Toutefois, cette manière de procéder se limite forcément à des collectivités spécifiques, où les relations entre les habitants sont très étroites. Elle présuppose, en outre, que les responsables des actes délinquants disposent de biens, ou sont en mesure de rembourser par le truchement de jours-travail des pertes qu'ils ont occasionnées. Il est évident, à l'opposé, qu'on ne saurait appliquer les mêmes modes de compensation dans le cadre des sociétés fortement industrialisées et dont le développement urbain impose l'anonymat ainsi que l'absence des relations qui prévalaient dans les contextes ruraux d'autrefois. La philosophie même qui est à la base des systèmes actuels de dédommagement des victimes a également changé. Autrefois, il s'agissait surtout de préserver une forme de bonne entente entre voisins, actuellement deux objectifs sont mis de l'avant.

En premier lieu les modalités de dédommagement, singulièrement quand il s'agit pour le coupable de travailler pour la victime, humanisent en quelque sorte le délinquant aux yeux de la personne lésée. Parallèlement, c'est un moyen de resocialiser l'individu coupable que de lui donner l'occasion de réaliser pleinement l'étendue de son acte, en fréquentant la victime. Il serait utopique, cependant, de considérer que cette forme de réparation, aussi moraliste puisse-t-elle paraître à certains, est applicable dans les cas de délits criminels impliquant des assauts physiques très graves. On imagine aisément à quel point il peut être difficile d'imposer aux victimes des viols, comme à celles qui sont devenues invalides à la suite d'une agression, sans parler du meurtre d'une personne proche, soit le père d'une famille ou, plus encore, un 
enfant, l'obligation pénible de fréquenter le responsabie. Certes des exceptions existent, mais quand on conçoit un système de dédommagement on est bien obligé de bâtir des modèles acceptables pour la majorité des concernés.

Le deuxième objectif du dédommagement des victimes repose sur l'idée d'atténuer l'impact de la criminalité et des réactions de vengeance éprouvées par l'opinion publique. C'est aussi exercer une action au niveau de la vindicte populaire à l'égard des délinquants trouvés coupables d'agressions physiques. C'est finalement accroitre tant soit peu le sentiment que la société offre une protection à ceux qui ont été victimisés. Certes, il est impossible au stade actuel des recherches de savoir dans quelle mesure les divers systèmes de dédommagements, tous relativement récents, atteignent leur but, nous allons donc nous contenter ici de les décrire dans leurs grandes lignes, puis de mentionner les modèles d'enquêtes entreprises au Canada.

\section{Les systèmes européens de dédommagement des victimes}

Historiquement, le premier cadre formel de dédommagement des victimes a été élaboré et voté en Nouvelle-Zélande en 1963, où le Tribunal de compensation des victimes d'actes criminels existe depuis 1964. En Europe, c'est en Grande-Bretagne que dès 1964 on a mis en pratique un programme de dédommagement des victimes, tandis que d'autres pays suivirent cet exemple après 1970, année au cours de laquelle le Comité européen pour les problèmes criminels du Conseil de l'Europe décida d'en entreprendre l'étude. Le Comité a examiné en premier lieu les insuffisances de recours dont disposent les victimes d'actes criminels, soit le procès civil qu'on peut intenter aux responsables de l'infraction, l'intervention en tant que partie civile dans le cadre du procès pénal, ainsi que certains moyens indirects.

Les conclusions qu'on peut en dégager sont valables dans tous les contextes et se résument à cinq constatations principales. Tout d'abord dans plusieurs cas le délinquant n'est pas identifié et à plus forte raison retrouvé. S'il est arrêté et jugé, il est généralement insolvable, ce qui signifie qu'un procès civil en vue d'obtenir des dommages-intérêts, impose des frais, des délais et fort peu de perspectives de réussite tangibles autre que la satisfaction purement symbolique. En ce qui a trait aux moyens qualifiés dans le 
rapport du Conseil de l'Europe comme * indirects ${ }^{1}$ on entend sous ce terme toute ordonnance incluant l'obligation pour le condamné de restituer à la victime les biens volés, ou encore de verser une amende prévue par le tribunal, exécuter des travaux spécifiques et autres, afin que la victime puisse être dédommagée. Or, tous ces moyens risquent fort d'être inopérants puisqu'ils présupposent au départ que le délinquant dispose des biens, revenus, ou capacité de travailler, ou d'un salaire ou revenu quelconque ce qui est en pratique plutôt rare. $\AA$ partir de ces conclusions le Comité a étudié les diverses formules d'indemnisation, dont l'assurance privée.

A cet égard, en Suède par exemple, existe une assurance contre les voies de fait, pour laquelle les primes sont très faibles si elle est contractée dans le cadre de l'assurance du chef de famille. Étant donné que selon les statistiques recensées, $90 \%$ de Suédois sont couverts par ce genre d'assurance, il est évident que les frais pour les concernés sont plus bas que ce ne serait le cas dans un pays où on voudrait l'introduire progressivement. La plupart des législations excluent en outre de l'assurance de responsabilité civile les conséquences des actes posés volontairement. Une victime de viol qui a fréquenté l'homme auparavant sans se douter qu'il va user à son égard de la force, sera donc automatiquement exclue et le même problème peut se poser au niveau des divers autres comportements qu'on peut qualifier par exemple d'imprudence, ou d'insouciance évidente. En Autriche, on $a$ intégré l'indemnisation des victimes dans le cadre de la sécurité sociale, ce qui permet de compenser les concernés pour la perte de période de travail, de leur garantir des soins médicaux gratuits, ou encore de prendre en charge ceux qui ont perdu le membre de la famille qui était son soutien. Il n'en reste pas moins que cette façon de procéder exclut les compensations pour la peine subie, évaluées par les tribunaux comme pretium doloris.

Toutes ces considérations ont permis au Comité de dégager les recommandations qui ont été suivies depuis, dans plusieurs pays, ce qui ne signifie pas que les propositions relatives à certaines normes ont été réalisées en Europe, aux États-Unis ou chez nous. Bien au contraire il semble, comme nous allons le préciser

1. Rapport final d'activités sur le dédommagement des victimes d'actes criminels. Conseil de l'Europe, Strasbourg, 1977, Doc. No. D.P.C./C.E.P.C. XXIX (77) 3 . 
plus loin, qu'en fait le dédommagement des victimes demeure très limité, compte tenu de l'importance des pertes réelles subies par certains individus et familles.

Quoi qu'il en soit les recommandations principales du Comité furent les suivantes :

1. Lorsque la réparation ne peut être assurée à un autre titre l'Etat doit contribuer à l'indemnisation de : a) toute personne ayant subi de graves lésions corporelles résultant d'une infraction; b) tous ceux qui étaient à la charge de la personne tuée lors d'une infraction.

2. En ce qui concerne l'infraction pénale génératrice du préjudice corporel, tous les actes de violence intentionnels devront au moins être couverts, même si l'auteur ne peut pas être poursuivi.

3. Le dédommagement peut se réaliser soit dans le cadre de la sécurité sociale, soit par l'institution d'un régime spécifique d'indemnisation, soit par le recours à l'assurance.

4. Le dédommagement doit être le plus complet et le plus équitable possible, prenant en considération la nature et les conséquences du préjudice.

5. Le dédommagement doit s'étendre selon le cas, au moins à la perte du revenu antérieur et futur, à l'augmentation des charges, aux frais médicaux, aux frais de rééducation médicale et professionnelle, ainsi qu'aux frais funéraires.

6. Pour des raisons pratiques ou économiques, le dédommagement peut comporter un minimum ou un maximum. Il peut aussi être fixé de façon forfaitaire en fonction du taux d'incapacité et de barèmes. L'attribution d'une indemnité peut être limitée aux victimes placées dans une situation matérielle grave.

7. Le dédommagement peut prendre la forme d'un capital ou d'une rente ${ }^{2}$.

8. Le dédommagement doit prévoir la possibilité d'accorder dans les cas urgents, une provision lorsque la détermination de l'indemnité risque de subir des retards.

Nous ne reproduisons ici que les recommandations qui nous apparaissent les plus importantes pour l'analyse ultérieure des régimes de dédommagement, tels qu'ils existent actuellement. $\mathrm{Ce}$ qu'il convient de retenir en résumé, dans les recommandations

2. Voir les pages 22 à 28 du rapport déjà cité. 
non citées, c'est le fait que le Comité estime que la victime doit garder le droit de poursuivre le délinquant dans tous les cas où le préjudice subi n'a pas été entièrement dédommagé. Mentionnons finalement la recommandation 11, selon laquelle : «Le dédommagement peut être réduit ou supprimé en tenant compte de l'attitude de la victime et de ses relations avec l'auteur et son milieu. »

L'examen des recommandations du Comité permet de relever plusieurs difficultés relatives à l'élaboration des politiques des dédommagements des victimes. Comment doit-on évaluer en effet les pertes du revenu futur en tenant compte des facteurs tels que la peur d'une nouvelle agression, par exemple ? Or, il est fréquent que les personnes qui ont été agressées en se rendant, ou en revenant de leur travail, sont incapables par la suite de surmonter certaines angoisses. D'autres ne parviennent pas à demeurer dans le même logement, ou maison, d'autres encore ne sont plus capables d'exercer leur métier et doivent se recycler. Dans tous ces cas, il est pratiquement impossible d'évaluer à priori les pertes réelles et on doit attendre plusieurs mois parfois pour être en mesure de disposer de certaines précisions. Si on désire parallèlement accorder une aide immédiate à la victime, il faut disposer, en somme, d'une caisse de dépannage d'autant plus difficile à administrer que l'évaluation des cas n'est guère automatique. Rien d'étonnant dès lors que dans certains pays on a préféré inclure les modes de dédommagements des victimes dans le cadre des programmes d'assurance médicale et sociale s'appliquant à l'ensemble de la population, ou encore aux catégories spécifiques des économiquement faibles.

Il y a également contradiction entre la première recommandation du Comité et les réserves faites en ce qui a trait à l'* attitude de la victime », ou encore « ses relations avec l'auteur du délit et son milieu $\$$. Contradiction fondamentale, à notre sens, puisqu'on la retrouve au niveau de l'ensemble des programmes de dédommagement des victimes.

Une victime qui refuse de coopérer avec la police par crainte de représailles de la part de son agresseur peut être considérée comme ayant une «attitude négative ». Doit-on, en toute justice sociale, refuser l'indemnité à une victime parce qu'elle a peur? En ce qui a trait aux relations de la victime avec l'auteur d'une agression, il est évident qu'on ne peut assumer le risque de 
dédommager un membre de la pègre sous le fallacieux prétexte qu'il a été maltraité par ses comparses, mais le problème de la violence à l'intérieur d'une famille, d'un groupe ou d'une communauté, demeure entier.

Une femme attaquée par son concubin, est une victime. Doit-on lui refuser le dédommagement parce qu'elle a mal choisi son concubin ou, au contraire, le lui accorder parce que ses moyens de défense étaient inférieurs, compte tenu de la force physique de l'homme?

Le problème est encore plus complexe lorsqu'il s'agit des enfants qui sont des victimes toutes désignées de certains parents cruels et dénaturés. C'est ainsi que l'enfant maltraité physiquement devrait théoriquement avoir droit à un dédommagement de l'Etat pour les pertes subies en raison des comportements de ses parents. Il est lié à eux par le hasard de sa naissance, ce qui signifie qu'il n'a pas la responsabilité de la relation qui existe entre lui et son père, par exemple. Or, les dommages des coups et sévices reçus dans l'enfance peuvent être irréversibles et multiples, mais leur évaluation demeure quasi impossible. Pour poser une question ad absurdum disons qu'on ne saura jamais ce que serait devenu Einstein, s'il avait été maltraité physiquement dans son enfance par un père ou une mère abusifs.

Rien d'étonnant dès lors que les programmes d'indemnisation varient selon les pays, mais qu'aucun ne peut être considéré comme susceptible de couvrir toutes les catégories des victimes d'actes définis en droit comme étant criminels.

D'une manière générale on tient compte des normes dont on trouve la formulation dans les recommandations du Conseil de l'Europe, mais les organes décisionnels sont soit judiciaires, soit administratifs, avec ou sans droit d'appel devant un tribunal. C'est ainsi qu'en Autriche comme en Allemagne fédérale les victimes doivent adresser leurs demandes d'indemnisation aux bureaux d'aide aux invalides et de victimes de guerre qui décident des suites à leur donner, tandis qu'en Belgique c'est un magistrat d'une juridiction spéciale qui statue. En France, l'indemnisation des victimes d'actes criminels relève des commissions qui sont des juridictions civiles composées de trois conseillers de la Cour d'appel, tandis qu'au Danemark c'est une commission formée d'un 
juge, d'un spécialiste en droit social et d'un avocat qui assume ce rôle.

Compte tenu du fait que dans ces pays le régime de dédommagement des victimes d'actes criminels n'a été institué qu'après 1970 (soit en Autriche, par exemple, en 1972, tandis qu'en France en 1977 uniquement), il est impossible de faire des comparaisons valables en vue d'analyser les avantages ou les désavantages respectifs de structures judiciaires par rapport aux structures administratives. Nous nous contentons donc de mentionner que cette dichotomie est liée aux deux courants de pensée. Le premier assimile les problèmes du dédommagement des victimes d'actes criminels à l'ensemble des mesures sociales, le deuxième, par contre, l'envisage comme une intervention judiciaire parfaitement distincte de toute forme d'aide sociale que l'État assure aux classes désavantagées ou à certains groupes spécifiques de citoyens. A la limite de ces deux approches, purement sociale et judiciaire, se situent les systèmes qu'on peut définir comme mixtes puisqu'il s'agit des commissions indépendantes sur lesquelles siègent des juges et des non juristes et dont le fonctionnement ne s'apparente pas nécessairement à celui d'un tribunal.

\section{Les systèmes de dédommagement des victimes aux États-Unis}

Le fait qu'on choisit une structure de préférence à une autre, est lié non seulement à l'ensemble du système d'aide sociale et d'action judiciaire qui prévaut dans un pays donné, mais aussi à la philosophie qui a prédominé au moment de l'adoption du régime de dédommagement. C'est ainsi qu'en Europe il s'agissait soit d'une très ancienne tradition britannique, soit de la recherche d'une nouvelle forme de justice sociale selon laquelle on a voulu reconnaître des droits à la victime, sachant fort bien qu'ils sont fort limités sur le plan légal. Dans les cas notamment où l'agresseur n'est pas connu, la victime d'un acte criminel qui le rapporte est bien obligée de se soumettre aux interrogatoires de la police pour aider la justice, mais ne peut espérer en contrepartie aucune reconnaissance tangible des risques qu'elle encourt parfois en le faisant. Par conséquent le dédommagement n'est pas uniquement un moyen de compensation, fort partiel d'ailleurs, puisque l'indemnisation ne saurait effacer ni la souftrance, ni la peur, mais aussi une forme d'incitation visant à encourager les citoyens à 
collaborer avec la police dont les taux de solutions de crimes baissent dangereusement dans plusieurs pays, ou régions.

La tradition américaine est fort différente. En effet, comme le rapportent Herbert Edelhertz et Gilbert Geis, dans leur excellent ouvrage sur le dédommagement des victimes ${ }^{3}$, il s'agissait en premier lieu d'offrir une forme de récompense au Bon Samaritain qui a été attaqué en portant secours à d'autres qui ont été agressés devant lui, sans que la police puisse intervenir. C'est là une philosophie qui reflète des tendances particulières, qu'on décèle dans les collectivités américaines et qui vont bien au-delà du sujet de cet article. À l'époque actuelle où existe un vacuum d'idées généreuses et où il est difficile de mobiliser les gens pour une cause humanitaire, autrement qu'en termes de manifestations, l'héröisme devient une denrée de plus en plus rare. On a supprimé les distinctions et les médailles, si chères encore au cour des Européens, et on qualifie plus aisément d'imbécile celui qui se tue pour sauver un autre et qui n'a pas su bien évaluer ses forces, que d'être généreux qui a obéi à une impulsion saine.

L'histoire vaut la peine d'être rapportée ici, bien qu'il ne s'agisse que d'un fait divers, puisqu'elle refiète, à notre sens un état d'esprit et aussi cette efficacité très américaine qui permet, en très peu de temps de mettre en pratique des réformes demandant ailleurs plusieurs années de réflexions, de discussions et de consultations. Le 9 octobre 1965, Arthur F. Collins, âgé de 28 ans, a été tué sous les yeux de sa femme qui a assisté impuissante au drame avec ses deux bébés dans les bras. Ils se trouvaient dans le métro, où un homme ivre s'est attaqué aux vieilles dames tranquillement assises dans leur coin. Arthur F. Collins est intervenu et a réussi à faire descendre l'agresseur, mais, à la dernière minute avant le départ du train, il est remonté et l'a tué à coups de couteau. La veuve et les enfants restaient sans autres ressources qu'une faible pension à laquelle Collins avait droit en tant que jeune programmeur à la compagnie Pan American. Deux jours après cet incident, John J. Gilhooley, président du Conseil des services du transport, réclamait une législation permettant le dédommagement des familles des victimes qui ont été tuées en essayant de protéger les autres. Le 23 novembre de la même année, c'est-à-dire cinq semaines plus tard, le

3. Public Compensation to Victims of Crime, par Herbert Edelhertz et Gilbert Geis, Editions Praeger Publishers Inc., 1974. 
Conseil de la ville de New York votait un règlement à cet égard, connu sous le nom de New York City Good Samaritan Measure $\triangleright$ et mis en pratique dès décembre. La veuve d'Arthur $F$. Collins a reçu en vertu de ce règlement une rente annuelle payable jusqu'à sa mort transmissible à sa fille si son décès devait se produire avant que cette dernière n'atteigne sa dix-huitième année.

Parallèlement les deux associations privées, le New York Republican Club et la Correctional Association of New York, ont commencé une action en vue de promouvoir l'adoption d'une législation au niveau de l'État. Un rapport de recherche sur le dédommagement des victimes venait juste d'être publié par le Comité des affaires municipales de New York et il contenait des statistiques susceptibles d'émouvoir l'opinion publique. En effet, le Bureau fédéral d'enquêtes (F.B.I.) y rapportait qu'en 1964, parmi les victimes des meurtres, on relevait un homme pour trois femmes, que l'âge de la plupart se situait entre 20 à 30 ans, ce qui permet de présumer que plusieurs ont laissé des enfants en bas âge ou autres personnes à charge. L'étude comparative des systèmes déjà en vigueur en Grande-Bretagne et en Nouvelle-Zélande, permettait en outre une évaluation de leur fonctionnement et l'élaboration des prévisions budgétaires.

Le gouverneur de l'État de New York a créé néanmoins un comité, chargé d'entendre les témoignages et de recevoir les mémoires de toutes personnes ou organismes intéressés, qui a commencé à tenir des audiences publiques dès janvier 1966.

Assez curieusement parmi ceux qui ont le plus sévèrement critiqué le projet on relève le témoignage d'un criminologue et juriste distingué, le professeur Gerhard O.W. Mueller. Il justifiait sa position en prétendant qu'une législation concernant le dédommagement des victimes risque d'accroître la criminalité de violence. En se basant sur les recherches faites dans le domaine des crimes contre la propriété, le professeur Mueller affirmait que les malfaiteurs ont moins de scrupules à s'attaquer aux grandes compagnies qu'aux biens des citoyens privés, parce qu'ils savent qu'elles sont de toute façon assurées et recevront donc une compensation. En deuxième lieu, il citait les travaux d'un autre criminologue américain, le professeur Marvin E. Wolfgang de l'Université de Pensylvanie, dont les recherches ont abouti aux conclusions que $70 \%$ d'homicides sont commis entre amis, amants, ou maris et 
femmes. A partir de ces données il prétendait, comme le fait également Marvin E. Wolfgang, qu'il s'agit fort souvent de la réaction du meurtrier à la provocation de la victime, réaction qui risque d'être d'autant plus facilitée que l'assassin saura que la famille de celle ou de celui à qui il s'attaque recevra un dédommagement ${ }^{4}$.

C'est un officier de police, Arthur Cornelius Jr,, qui a attaqué le témoignage du distingué professeur d'université en affirmant ce qui suit :

I have heard the observation that a compensatory plan might have a worsening effect on the crime picture in that the criminally oriented would be encouraged to acts of violence because of the knowledge that victims would not suffer financially. I reject this view. I think it attributes qualities to criminals they do not possess. If the criminal were really sensitive about the effects of his activity, it is impossible he would be a criminal.

Il nous semble important de compléter cette constatation découlant du bon sens fort élémentaire d'un officier de police par deux constatations. En premier lieu, les études de Marvin E. Wolfgang s'appliquent probablement à certains groupes ou encore à certaines époques fort éloignées, mais l'actualité quotidienne les dément à travers les faits divers rapportés par les journaux. Les personnes âgées attaquées dans la rue, dévalisées, brutalisées et parfois tuées, sont des victimes en puissance des bandes de jeunes qui opèrent dans les grands centres urbains. La même constatation s'applique aux petits commerçants dont traite, dans cette livraison de la revue, Micheline Baril, ou encore aux adolescents assassinés par des adultes malades mentaux, obsédés sexuels, ou autres.

En deuxième lieu, le concept même de la "provocation 》, dont une victime, une femme singulièrement, peut se rendre coupable, est fort imprécis. Si on prend pour acquis que nous vivons en Occident dans des sociétés civilisées, le fait qu'une jeune fille demande à un conducteur de la prendre à bord d'un véhicule automobile signifie qu'elle sollicite un service et non pas un viol. La perception de cette sollicitation ne dépend pas, en somme de la conduite de la fille en question, mais du degré de socialisation du conducteur concerné. Il est assez significatif qu'on ne discute que

4. Pour détails, voir l'ouvrage déjà cité, pages 29 et 30. 
rarement de la «provocation \$ de la victime, quand il s'agit d'un homme placé dans la même situation, bien que le conducteur peut en principe être un homosexuel en mal de conquête ayant pour objet une personne du même sexe que lui. En d'autres termes le concept de «provocation » de la victime semble être étroitement lié à la nature des rapports de forces. La femme étant plus faible physiquement que le mâle et le vieillard qu'un homme en pleine possession de ses muscles et biceps, on peut toujours accuser dans ce contexte la victime d'avoir été provoquante. Le fait qu'un homme âgé se promène dans la rue et se fait tuer par un agresseur qui désire défouler son agressivité ou s'emparer de son portefeuille peut être retenu dès lors en cour comme une forme de provocation et un élément atténuant la responsabilité du dit agresseur.

Déjà, dans certaines causes existent des précédents. On reproche aux victimes d'avoir refusé à l'agresseur l'argent demandé, ou encore d'avoir essayé de se défendre, ce qui pose en termes bien contestables le problème de la responsabilité de la victime. Il n'est pas de notre propos d'analyser ici cette question, déjà traitée par E. Fattah, mais il nous semble utile de souligner qu'il est naturel à l'individu, même âgé, même de sexe féminin, d'avoir des réflexes de défense face à une agression.

Le Comité créé par Nelson Rockefeller, gouverneur à l'époque de l'État de New York, a entendu également un autre témoignage particulièrement intéressant à notre avis. Il s'agissait du cas concernant l'aide apportée à la police par un citoyen. En effet, Arnold Schuster a informé les autorités d'avoir vu Willie Sutton, un homme recherché par le F.B.I., changer le pneu de sa voiture dans le voisinage de son lieu de travail. Grâce à ces indications, Willie Sutton a été arrêté, mais on a annoncé parallèlement dans la presse l'importance de l'intervention de Schuster dans cette affaire. Pendant un certain temps, ce dernier a été protégé par la police, puis les gardes de corps ont été retirés et peu après il a été assassiné par la pègre pour avoir facilité l'arrestation d'un de ses membres.

Les héritiers de la victime ont entrepris des poursuites contre la Ville de New York, en alléguant que la protection qu'on lui avait assurée était insuffisante. La cour d'appel a statué en faveur des plaignants, mais le jugement était rédigé de façon à préciser 
qu'il s'agit d'un cas d'espèce qui ne doit pas être considéré comme un précédent en droit ${ }^{5}$.

En somme cette cause n'a pas inspiré la philosophie de la législation relative au dédommagement des victimes d'actes criminels, mais principalement l'idée force de récompenser, en partie tout du moins, la famille du «Bon Samaritain ».

Le projet de loi présenté dès avril 1966, soit six mois à peine à compter du meurtre d'Arthur Collins et adopté au mois de mai de la même année, a institué une commission formée de trois avocats ayant un minimum de dix ans d'expérience et devant être employés à temps plein. Le Crime Victims Compensation Board est un organisme appelé à statuer dans tous les cas de victimes des crimes commis dans l'État de New York. Ses décisions peuvent être contestées en appel, mais la victime entendue par un des commissaires, peut demander, si elle s'estime lésée, que son cas soit révisé en premier lieu par les trois membres du Comité spécialement réunis à cet effet. Les membres de la Commission sont nommés pour sept ans et constituent une sorte de tribunal administratif. La loi précise qu'ils sont autorisés à accorder un dédommagement aux victimes de tout acte criminel, y compris ceux dont l'auteur n'est pas connu, ou n'a pas été appréhendé, mais que la compensation maximale ne peut dépasser $\$ 15000$. II n'est donc pas question ici d'appliquer les recommandations du Conseil de l'Europe, selon lesquelles il convient de prévoir dans certains cas des pensions annuelles, totalisant des sommes beaucoup plus élevées. Plus encore, si la veuve de Collins n'avait pas reçu préalablement sa pension, sous l'empire de la loi, elle n'aurait pu compter que sur une aide infiniment plus limitée que ce ne fut son cas.

Au cours des années 1973, on a amendé la loi en élargissant le droit à la compensation à des membres de la famille autres que les conjoints et les enfants. Le principe de dédommagement pour les conséquences de n'importe quel genre de délit est resté inchangé. Cela signifie que la loi s'applique autant aux victimes des crimes de violence qu'à ceux d'une fraude, même anticipée, mais à ce niveau les statistiques démontrent que le nombre de demandes est très limité. En ce qui a trait aux crimes étant la conséquence d'un conflit à l'intérieur de la famille, la loi stipule que la

5. Pour détails, voir l'ouvrage déjà cité, pages 30 et 31 . 
famille du délinquant $n$ 'a pas droit à un dédommagement. Dans certains cas, toutefois, contrairement aux principes qui prévalent théoriquement dans l'élaboration des législations européennes et américaines en la matière, si le père a tué la mère, le Comité peut accorder une compensation aux enfants et des précédents existent à cet égard, surtout dans les cas où le couple a été séparé de fait, ou de jure.

Le principal reproche qu'on adresse au législateur de l'État de New York, c'est d'avoir, pour des raisons d'économie, imposé des normes financières dans le cadre desquelles peut être versé un dédommagement à la victime. Le concerné doit faire preuve, en effet, qu'il se trouve dans des conditions économiquement difficiles, ce qui signifie finalement que seuls les milieux désavantagés ont droit à une compensation, à l'exclusion de la classe moyenne et des bien nantis. Or plusieurs, dont le professeur Robert Childres, prétendent que le système de compensation des victimes doit s'appliquer à tous les citoyens, et non pas uniquement à ceux qui ont des revenus et salaires en deçà de la moyenne nationale. Selon Childres, professeur à la faculté de droit de Northwestern University :

Debilitating poverty is a blot on the national character of any prosperous nation. For most poverty, however, there is no admitted causal relationship involving the government. For destitution threatened by criminal injury to the person, there unquestionably is such a relationship. Victims of crime ought not be required to divest themselves of all resources before qualifying for compensation. Nor should they receive payments at a level kept low in part to induce people to return to the work force ${ }^{6}$.

Il n'en reste pas moins que la loi n'a pas été changée à cet égard et qu'elle s'adresse principalement aux économiquement faibles, tout en maintenant un régime de compensation limité à $\$ 15000$. Elle est appliquée par une sorte de tribunal administratif, le Crime Victims Compensation Board sur lequel siègent des avocats ayant plusieurs années d'expérience. En pratique, il ne s'agit pas de dédommagement des victimes d'actes criminels, mais bien davantage de la reconnaissance d'un principe permettant de verser des compensations aux victimes qui représentent sur le plan économique les éléments les plus faibles dans la société.

6. Voir livre déjà cité, pages 32 et 33 . 
La situation dans les autres états américains, où existe un régime de dédommagement des victimes est comparable à celle qui prévaut dans l'État de New York. C'est ainsi qu'en Californie la loi d'indemnisation des victimes d'actes criminels a été introduite en 1965 à la suite de l'intervention d'un juge, tandis qu'au Massachusetts, une commission a été nommée pour étudier le problème. Dans d'autres états, tel le Nevada on a mis de l'avant en premier lieu le principe du Bon Samaritain que la société a l'obligation de reconnaître comme méritant d'être compensé pour son courage. Généralement, toutefois, le maximum de dédommagement a été fixé à $\$ 15000$, ou encore à $\$ 10000$. L'État de Hawaï constitue à cet égard une exception puisqu'on y a rejeté toutes les restrictions concernant le niveau des revenus et l'état de fortune de la victime. En Californie l'application de la loi est administrée avec un succès mitigé par des services sociaux tandis qu'au Massachusetts les victimes doivent présenter leur demande aux cours municipales, ou cours de districts. En somme, il ne s'agit pas d'un modèle commun à l'ensemble du pays, mais plutôt d'approches diverses et de modalités de mise en application très variables. Il n'en reste pas moins qu'on peut distinguer trois constantes. Tous les programmes y compris celui du gouvernement fédéral, maintiennent une distinction entre les droits de ceux qui sont devenus victimes en voulant venir en aide à un tiers et les autres. Les Bons Samaritains, comme on les définit en droit, peuvent recevoir une compensation sans qu'on tienne compte de leurs revenus tandis que pour les victimes en général c'est la règle. Au niveau de l'application il y a toujours un maximum de dédommagement soit un montant qui ne peut pas être dépassé. Les victimes doivent déposer leurs demandes tantôt auprès d'une commission spéciale, un service social, ou une cour, mais conservent le droit d'aller en appel devant un tribunal supérieur.

\section{Le dédommagement des victimes au Canada}

A l'instar des législations de certains états américains, le Code criminel canadien prévoit également la possibilité pour la victime de demander un montant comme réparation ou dédommagement pour la perte de biens ou le dommage à des biens qu'a subi le requérant par la suite de la perpétration de l'infraction » (Art. 653, C.C.). Toutefois, au Canada, comme aux Etats-Unis et en Europe, les régimes de dédommagement des victimes ont été institués en vue de compenser les concernés bien plus pour les 
dommages physiques que matériels. Par ailleurs, comme nous l'avons déjà mentionné, les responsables ne sont pas toujours connus et parmi ceux qui ont été arrêtés et jugés, le pourcentage d'individus susceptibles d'assurer un remboursement ou une réparation est très faible. En somme, au Canada la situation est la même qu'ailleurs et les victimes ne peuvent compter que rarement sur la possibilité d'être dédommagées par l'auteur du délit. Toutefois, en raison des services disponibles la victime des actes criminels a accès à plusieurs ressources et cela au même titre que tous les autres citoyens. L'assurance-maladie couvre les frais de l'hospitalisation, l'assurance-chômage rembourse une partie du salaire qui n'a pu être gagnée, les pensions d'invalidité sont versées à tous ceux qui peuvent faire la preuve d'y avoir droit et les personnes n'ayant pas de revenus sont éligibles pour obtenir l'assistance sociale. Ceux, en outre, qui ont des assurances privées, sont dédommagés en cas de vols ou de dommages, selon les normes prévues dans leurs polices.

La philosophie qui a été à l'origine de l'établissement du système de dédommagement des victimes au Canada n'est pas liée uniquement au problème d'apporter une aide financière à la victime d'actes criminels. Ses objectifs sont triples. En premier lieu, il s'agit d'inciter ainsi les citoyens à rapporter les actes criminels qui sans leur aide ne serait pas signalés selon toute probabilité. En deuxième lieu, on espère que les gens vont plus volontiers collaborer avec la police sachant que la collectivité reconnaît leur droit à la compensation. En troisième lieu, il s'agit d'une forme d'appréciation des actes posés par des citoyens qui interviennent pour prévenir un crime ou pour aider à arrêter le responsable. D'une façon plus globale, il convient de reconnaître également que sur le plan social et politique, le dédommagement des victimes a plusieurs avantages, dont celui d'atténuer les réactions d'hostilité à l'égard des criminels incarcérés, logés et nourris aux frais des contribuables, contrairement à la victime qui, elle, doit subvenir à ses besoins. Dans les campagnes visant à supprimer la peine capitale, le fait d'instaurer les régimes de compensation des victimes, n'a certes pas eu d'efiet prépondérant, mais représentait un élément de persuasion valable.

À l'opposé, les inconvénients de ces régimes sont fort limités en pratique. En dehors de ceux, déjà mentionnés au préalable on a invoqué chez nous la possibilité de fraude, autant de la part des 
victimes des actes d'agression physique exercés à l'intérieur de la famille, que des comparses des criminels, impliqués dans un délit, mais non identifiés comme complices par les services policiers. Toutefois, ce genre d'abus relève des contrôles instaurés par les organismes chargés de distribuer les dédommagements et ne saurait remettre en cause le principe même qui a présidé à leur création.

Au Canada, seulement deux provinces sur dix n'ont pas instauré jusqu'à présent de système de compensation des victimes d'actes criminels, soit l'île du Prince Édouard et la NouvelleÉcosse. La compensation est prévue pour les dommages causés à la personne à la suite d'un acte de violence, mais non pas en tant que remboursement des biens volés. Le niveau de la compensation est limité par un maximum, qui est toujours plus élevé pour les victimes qui ont été attaquées en essayant de défendre ou de protéger des tiers. Dans leur cas s'applique le dédommagement pour la «douleur et les souffrances», ce qui n'est pas accordé nécessairement aux autres catégories des victimes. Dans la majorité des provinces on exclut, en outre, le dédommagement des victimes vivant avec leur agresseur. En somme un enfant maltraité par un père brutal ou incestueux, n'a pas le droit de réclamer l'indemnisation en tant que victime, ce qui constitue en fait une grave injustice. On justifie cette façon de procéder par le souci d'empêcher l'agresseur, le père en l'occurrence, ou encore le mari qui brutalise sa femme, de profiter indirectement de l'argent ainsi obtenu. II n'en reste pas moins qu'objectivement c'est là une source d'injustice à l'égard des femmes et des enfants maltraités qui ont la possibilité d'obtenir une aide sociale, mais non pas le droit de réclamer une compensation.

Par ailleurs, dans le cadre du régime d'indemnisation canadien existe le concept de l'«indignité » de la victime. C'est ainsi que selon la législation du Manitoba on peut tenir compte dans l'évaluation des droits de la victime à la compensation non seulement de son comportement, mais encore de sa moralité. C'est là, croyons-nous, un principe fort discutable. Dans les cas de viol notamment, le fait que la victime a été consentante par le passé à avoir des relations avec plusieurs hommes, peut l'empêcher de réclamer un dédommagement. Or, on voit mal pourquoi il convient de permettre cette forme d' "évaluation», de la moralité de la victime puisqu'on ne la dédommage pas en principe en raison de 
sa personnalité, mais en fonction de la soufirance physique qui lui a été infligée.

En ce qui a trait à l'application des régimes de dédommagement, elle relève des commissions spéciales, qui sont en fait des tribunaux administratifs, et c'est le cas pour l'Ontario, l'Alberta, Terre-Neuve, Manitoba et la Saskatchewan. Au Québec et en Colombie-Britannique, ce sont des Commissions des accidents de travail ${ }^{7}$ qui assurent la responsabilité de recevoir les demandes des victimes et de statuer. Soulignons à ce propos que la législation québécoise est particulièrement intéressante et apparaît comme plus équitable que certaines législations américaines. En effet, selon l'article 5 de la Loi de l'indemnisation des victimes (S.Q. chap. 18) :

Les avantages dont peuvent bénéficier suivant la présente loi, la victime d'un crime ou ses dépendants sont les bénéfices prévus aux sections III, IV et V de la Loi des accidents du travail (Statuts refondus, 1964, chapitre 159).

Il peut en outre être accordé à la mère qui pourvoit ellemême à l'entretien d'un enfanti né par suite d'un viol, pour l'entretien de cet enfant, une rente mensuelle égale à la rente accordée, suivant la Loi des accidents du travail, à une veuve ayant un enfant. Si la mère décède, la rente peut être accordée à toute personne compétente qui prend soin de cet enfant à la satisfaction de la Commission.

Nous avons discuté en détails de la philosophie, comme de l'évolution historique, du principe du dédommagement des victimes d'actes criminels, nous allons donc nous contenter, en ce qui a trait au système canadien, de le traiter surtout à travers l'analyse des statistiques illustrant son importance selon les provinces ${ }^{8}$.

Sur la base des tableaux qui précèdent on constate que l'application du régime d'indemnisation des victimes d'actes criminels date de 1973 pour six provinces et de 1976 pour l'Alberta, le Québec, les Territoires du Nord-Ouest et le Yukon. Étant

7. Pour détails concernant les divers régimes et singulièrement celui de l'Ontario, voir $\&$ Dédommagement et indemnisation des victimes d'actes criminels et droit pénal canadien par Allen Linden, Commission de réforme du droit du Canada, 1976.

8. Nous tenons à remercier tout particulièrement $\mathbf{M}$. David $\mathbf{M}$. Farrell, du Service de la planification et d'élaboration de la politique du ministère de la Justice du Canada qui a bien voulu nous faire parvenir les statistiques les plus récentes. 
TABLEAU I

Indemnisation des victimes dactes criminels

\begin{tabular}{|c|c|c|c|c|c|c|c|}
\hline \multirow[t]{2}{*}{ Province } & \multirow[t]{2}{*}{ Année fiscale } & \multirow[t]{2}{*}{$\begin{array}{l}\text { Nombre de } \\
\text { dédommagements } \\
\text { accordés }\end{array}$} & \multicolumn{2}{|c|}{$\begin{array}{l}\text { Total des indemnisations } \\
\text { versées dans le cadre de } \\
\text { l'accord fédéral/provincial }\end{array}$} & \multicolumn{2}{|c|}{$\begin{array}{l}\text { Contribution du } \\
\text { gouvernement fédéral }\end{array}$} & \multirow[b]{2}{*}{$\%$} \\
\hline & & & (Dollars) & (Per capita) & (Dollars) & (Per capita) & \\
\hline Colombie-Britannique & $\begin{array}{l}\text { Jan-Mars/73 } \\
1973-74 \\
1974-75 \\
1975-76 \\
1976-77 \\
1977-78\end{array}$ & $\begin{array}{r}31 \\
160 \\
278 \\
348 \\
450 \\
391\end{array}$ & $\begin{array}{r}\mathbf{5} 25556 \\
243577 \\
658334 \\
1011659 \\
1210794 \\
1180872\end{array}$ & $\begin{array}{l}011 \\
10 \\
27 \\
41 \\
49 \\
47\end{array}$ & $\begin{array}{r}\$ 21988 \\
115750 \\
119750 \\
122850 \\
124550 \\
249760\end{array}$ & $\begin{array}{l}0095 \$ \\
05 \\
05 \\
05 \\
05 \\
10\end{array}$ & $\begin{array}{l}86 \% \\
48 \\
18 \\
12 \\
10 \\
21\end{array}$ \\
\hline Alberta & $\begin{array}{l}\text { Jan-Mars/76 } \\
1976-77 \\
1977-78\end{array}$ & $\begin{array}{r}42 \\
241 \\
211\end{array}$ & $\begin{array}{r}76386 \\
411869 \\
388622\end{array}$ & $\begin{array}{l}04 \\
23 \\
20\end{array}$ & $\begin{array}{r}22100 \\
91300 \\
189970\end{array}$ & $\begin{array}{l}0125 \\
05 \\
10\end{array}$ & $\begin{array}{l}29 \\
22 \\
49\end{array}$ \\
\hline Saskatchewan & $\begin{array}{l}\text { Jan-Mars/73 } \\
1973-74 \\
1974-75 \\
1975-76 \\
1976-77 \\
1977-78\end{array}$ & $\begin{array}{l}13 \\
62 \\
59 \\
55 \\
60 \\
85\end{array}$ & $\begin{array}{r}19113 \\
154187 \\
116282 \\
124797 \\
168107 \\
165708\end{array}$ & $\begin{array}{l}02 \\
17 \\
13 \\
14 \\
18 \\
18\end{array}$ & $\begin{array}{l}11450 \\
45400 \\
45350 \\
45900 \\
46750 \\
82854\end{array}$ & $\begin{array}{l}013 \\
05 \\
05 \\
05 \\
05 \\
09\end{array}$ & $\begin{array}{l}60 \\
29 \\
39 \\
37 \\
28 \\
50\end{array}$ \\
\hline
\end{tabular}

* On remarquera que le pourcentage de la contribution fédérale au programme de dédommagement des victimes n'est pas fixe et varie selon les provinces et les années. 
TABLEAU I (suite)

\begin{tabular}{|c|c|c|c|c|c|c|c|}
\hline \multirow[t]{2}{*}{ Province } & \multirow[t]{2}{*}{ Année fiscale } & \multirow[t]{2}{*}{$\begin{array}{l}\text { Nombre de } \\
\text { dédommagements } \\
\text { accordés }\end{array}$} & \multicolumn{2}{|c|}{$\begin{array}{l}\text { Total des indemnisations } \\
\text { versées dans le cadre de } \\
\text { l'accord fédéral/provincial }\end{array}$} & \multicolumn{2}{|c|}{$\begin{array}{l}\text { Contribution du } \\
\text { gouvernement fédéral }\end{array}$} & \multirow[b]{2}{*}{$\% *$} \\
\hline & & & (Dollars) & (Per capita) & (Dollars) & (Per capita) & \\
\hline Manitoba & $\begin{array}{l}\text { Jan-Mars } / 73 \\
1973-74 \\
1974-75 \\
1975-76 \\
1976-77 \\
1977-78\end{array}$ & $\begin{array}{r}0 \\
51 \\
95 \\
92 \\
81 \\
90\end{array}$ & $\begin{array}{r}25400 \\
113936 \\
240163 \\
234579 \\
313142 \\
369225\end{array}$ & $\begin{array}{l}03 \\
11 \\
24 \\
23 \\
30 \\
36\end{array}$ & $\begin{array}{r}12400 \\
49900 \\
50550 \\
50950 \\
51400 \\
103130\end{array}$ & $\begin{array}{l}013 \\
05 \\
05 \\
05 \\
05 \\
10\end{array}$ & $\begin{array}{l}49 \% \\
44 \\
21 \\
22 \\
16 \\
28\end{array}$ \\
\hline Ontario & $\begin{array}{l}\text { Jan-Mars/73 } \\
1973-74 \\
1974-75 \\
1975-76 \\
1976-77 \\
1977-78\end{array}$ & $\begin{array}{r}86 \\
402 \\
349 \\
497 \\
604 \\
563\end{array}$ & $\begin{array}{r}133463 \\
730941 \\
727230 \\
899590 \\
1409291 \\
1623857\end{array}$ & $\begin{array}{l}02 \\
09 \\
09 \\
11 \\
17 \\
19\end{array}$ & $\begin{array}{r}97813 \\
396950 \\
404700 \\
411300 \\
416550 \\
811929\end{array}$ & $\begin{array}{l}013 \\
05 \\
05 \\
05 \\
05 \\
097\end{array}$ & $\begin{array}{l}73 \\
54 \\
56 \\
46 \\
30 \\
50\end{array}$ \\
\hline Québec & $\begin{array}{l}1975-76 \\
1976-77 \\
1977-78\end{array}$ & $\begin{array}{r}768 \\
999 \\
1039\end{array}$ & $\begin{array}{l}1950708 \\
2624119 \\
2713861\end{array}$ & $\begin{array}{l}32 \\
42 \\
43\end{array}$ & $\begin{array}{l}309400 \\
312150 \\
628310\end{array}$ & $\begin{array}{l}05 \\
05 \\
10\end{array}$ & $\begin{array}{l}16 \\
12 \\
34\end{array}$ \\
\hline Nouveau-Brunswick & $\begin{array}{l}\text { Jan-Mars } / 73 \\
1973-74 \\
1974-75 \\
1975-76 \\
1976-77 \\
1977-78\end{array}$ & $\begin{array}{r}2 \\
9 \\
8 \\
16 \\
12 \\
9\end{array}$ & $\begin{array}{r}7400 \\
30808 \\
22769 \\
83261 \\
37002 \\
30636\end{array}$ & $\begin{array}{l}011 \\
05 \\
03 \\
12 \\
05 \\
04\end{array}$ & $\begin{array}{r}6660 \\
27727 \\
20492 \\
33750 \\
33302 \\
27573\end{array}$ & $\begin{array}{l}0102 \\
043 \\
031 \\
05 \\
05 \\
04\end{array}$ & $\begin{array}{l}90 \\
90 \\
90 \\
40 \\
90 \\
90\end{array}$ \\
\hline
\end{tabular}




\begin{tabular}{|c|c|c|c|c|c|c|c|}
\hline \multirow{3}{*}{$\begin{array}{l}\text { Province } \\
\text { Terre-Neuve }\end{array}$} & \multirow{3}{*}{$\begin{array}{l}\text { Annte fiscale } \\
\\
\text { Jan-Mars/73 } \\
1973-74 \\
1974-75 \\
1975-76 \\
1976-77 \\
1977-78\end{array}$} & \multirow{3}{*}{$\begin{array}{c}\begin{array}{c}\text { Nombre de } \\
\text { dédommagements } \\
\text { accordés }\end{array} \\
0 \\
2 \\
3 \\
4 \\
1 \\
1\end{array}$} & \multicolumn{2}{|c|}{$\begin{array}{l}\text { Total des indemnisations } \\
\text { versées dans le cadre de } \\
\text { l'accord fédéral/provincial }\end{array}$} & \multicolumn{2}{|c|}{$\begin{array}{l}\text { Contribution du } \\
\text { gouvernement fédéral }\end{array}$} & \multirow{3}{*}{$\begin{array}{c}\% * \\
0 \% \\
90 \\
90 \\
90 \\
90 \\
90\end{array}$} \\
\hline & & & (Dollars) & (Per capita) & (Dollars) & (Per capita) & \\
\hline & & & $\begin{array}{l}0 \\
3385 \\
3761 \\
6824 \\
1534 \\
1422\end{array}$ & $\begin{array}{l}0 \\
01 \\
01 \\
01 \\
0 \\
003\end{array}$ & $\begin{array}{l}0 \\
3047 \\
3385 \\
6142 \\
1381 \\
1280\end{array}$ & $\begin{array}{l}0 \\
006 \\
006 \\
01 \\
0 \\
002\end{array}$ & \\
\hline $\begin{array}{l}\text { Territoire du } \\
\text { Nord-Ouest }\end{array}$ & $\begin{array}{l}1975-76 \\
1976-77 \\
1977-78\end{array}$ & $\begin{array}{l}7 \\
5 \\
2\end{array}$ & $\begin{array}{l}24163 \\
45633 \\
19172\end{array}$ & $\begin{array}{r}64 \\
1,20 \\
2,26\end{array}$ & $\begin{array}{l}18123 \\
34225 \\
14379 * *\end{array}$ & $\begin{array}{r}48 \\
90 \\
3,01\end{array}$ & $\begin{array}{l}75 \\
75 \\
75\end{array}$ \\
\hline Yukon & $\begin{array}{l}1976-77 \\
1977-78\end{array}$ & $\begin{array}{l}1 \\
1\end{array}$ & $\begin{array}{l}109 \\
959\end{array}$ & $\begin{array}{l}005 \\
04\end{array}$ & $\begin{array}{r}82 \\
719\end{array}$ & $\begin{array}{l}003 \\
03\end{array}$ & $\begin{array}{l}75 \\
75\end{array}$ \\
\hline Total & $\begin{array}{l}\text { Jan-Mars/73 } \\
1973-74 \\
1974-75 \\
1975-76 \\
1976-77 \\
1977-78\end{array}$ & $\begin{array}{r}132 \\
686 \\
792 \\
1829 \\
2454 \\
2392\end{array}$ & $\begin{array}{r}210932 \\
1276834 \\
1768539 \\
4411967 \\
6221600 \\
6494334\end{array}$ & $\begin{array}{l}009 \\
06 \\
08 \\
19 \\
27 \\
28\end{array}$ & $\begin{array}{r}150311 \\
638774 \\
644227 \\
1020515 \\
1111690 \\
2109904\end{array}$ & $\begin{array}{l}006 \\
03 \\
03 \\
04 \\
05 \\
09\end{array}$ & $\begin{array}{l}71 \\
50 \\
36 \\
23 \\
18 \\
32\end{array}$ \\
\hline
\end{tabular}

** Montant payé au cours de l'année fiscale 1979-80. 
TABLEAU II

Dépenses per capita relatives d̀ l'indemnisation des victimes d'actes criminels (Total des indemnisations versées dans le cadre de l'accord fédéral/provincial)

\begin{tabular}{llcc} 
& $1975-76$ & $1976-77$ & $1977-78$ \\
\hline Colombie-Britannique & 41 & 49 & 47 \\
Alberta & 04 & 23 & 20 \\
Saskatchewan & 14 & 18 & 18 \\
Manitoba & 23 & 30 & 36 \\
Ontario & 11 & 17 & 19 \\
Québec & 32 & 42 & 43 \\
Nouveau-Brunswick & 12 & 05 & 04 \\
Terre-Neuve & 01 & 00 & 003 \\
Territoires du Nord-Ouest & 64 & 1,20 & 2,26 \\
Yukon & - & 005 & 04 \\
\hline
\end{tabular}

donné que selon les recherches américaines deux principaux facteurs influent sur le nombre de demandes d'indemnisation; le taux de victimisation et le degré d'information dans le public en ce qui concerne l'existence d'un service de compensation; on pourrait présumer que ce sont les provinces où ces services existent depuis 1973 qui ont des taux de demandes les plus élevées.

Or, en pratique à partir des années $1975-76$, on note une augmentation sensible pour la Colombie-Britannique et l'Ontario, mais non pas pour la Saskatchewan et le Manitoba. En Alberta et au Québec, en cutre, où les régimes de compensation ont été introduits uniquement en 1976, la progression diffère sensiblement. En Alberta on constate une brusque montée en 1976-77, due probablement à l'introduction effective du régime de compensation, puis une baisse en 1977-78, tandis qu'au Québec on accuse une augmentation constante puisqu'on passe de 768 en 1975-76, à 999 en 1976-77, et à 1039 en 1977-78. La comparaison entre les données de la Colombie-Britannique et de l'Ontario, permet de déceler un certain plafonnement qui se solde par une baisse en 1977-78 observable dans les deux provinces.

En ce qui a trait aux coûts par habitant, ils sont les plus élevés pour la Colombie-Britannique, où ils atteignent $49 \varnothing$ en 1976-77. A cet égard, il est intéressant de mentionner que dans les Territoires du Nord-Ouest, qu'il convient de traiter séparément puisqu'il ne s'agit pas d'une province, ces coûts ont été en 1977-78 pratiquement cinq fois plus élevés qu'en Colombie- 
Britannique, soit un taux per capita de $\$ 2,26$, comparativement à celui de 47 cents.

Comment ces différences se justifient-elles? S'agit-il d'une plus forte criminalité de violence, ou d'une meilleure collaboration des victimes concernées avec les autorités, ou encore des normes de dédommagement plus généreuses? Étant donné le fait que les coûts per capita sont les plus élevés, depuis les trois dernières années pour la Colombie-Britannique et le Québec, il est loisible de présumer que les modalités d'attribution des dédommagements ont une influence à cet égard. En effet, c'est dans ces deux provinces que le système est confié aux Commissions des accidents du travail. L'étude des différences des montants accordés en termes de dédommagement semble confirmer cette hypothèse. C'est ainsi qu'en Alberta, par exemple, on a dépensé en 1977-78, la somme totale de $\$ 388622$ pour répondre aux 211 demandes, tandis qu'en Colombie-Britannique au cours de la même année, on a dépensé $\$ 1180872$ pour 391 demandes, soit presque trois fois plus.

Il n'en reste pas moins que le calcul des dépenses moyennes par demande n'a pas de signification en soit et qu'il convient d'étudier le genre d'agression et de dommages causés à la victime avant de pouvoir préciser si ces différences dépendent de leur importance, ou si elles sont dues principalement aux modes d'évaluation des organes distribuant les fonds.

\section{Conclusions}

Doit-on conclure que le meilleur système de dédommagement des victimes demeure celui dont l'application est confiée aux organismes l'assimilant à la compensation des accidents de travail ? Certes, contrairement aux autres services et tribunaux administratifs, les personnes qui siègent sur les Commissions des accidents du travail, sont généralement très soucieuses de privilégier, dans la mesure où la loi le permet, l'accidenté. On tient compte comme de bien entendu de la faute professionnelle, ou de l'imprudence commise au travail, mais il n'en reste pas moins qu'on a un préjugé favorable à l'égard d'un individu, blessé ou devenu invalide à la suite de l'exercice de son métier qui est en même temps son gagne-pain. En traitant le cas de la victime d'un acte criminel dans un tel contexte on évalue plus aisément la perte de jours, ou d'heures de travail, que ce n'est le cas pour les 
autres types de commissions, bien que théoriquement on se sert toujours des mêmes barèmes de base. Il n'en reste pas moins qu'il y a lieu de se demander si la Commission des accidents du travail n'a pas certaines diffcultés à comptabiliser par exemple les pertes encourues par les propriétaires des magasins, ces petits commerçants qui n'ont pas droit aux avantages sociaux prévus pour les employés et qui ne parviennent à gagner des revenus appréciables qu'en travaillant pendant un nombre d'heures beaucoup plus élevé. Au-delà des normes légales, se situent là des réactions difficilement mesurables de façon globale et qu'on ne peut évaluer qu'en analysant chaque dossier à son propre mérite.

Or, tous les systèmes de dédommagement des victimes procèdent des idées généreuses, mais leur importance réelle dépend bien plus de leur application que de la philosophie qui a été à l'origine de l'élaboration de la loi. Les nécessités de recherches en la matière demeurent dès lors d'autant plus urgentes que dans tous les pays où de telles mesures ont été adoptées, il $s$ 'agit des procédures relativement récentes, souvent mal connues du grand public, et qu'il convient d'évaluer pour se rendre compte si elles correspondent pleinement aux objectifs visés et aux buts qu'on veut atteindre.

\section{BIBLIOGRAPHIE}

CABINET DU MINISTRE DE LA JUSTICE : Communiqués, 28 déc. 1972, 15 mars 1973, 10 mai et 24 mai 1973, Ottawa, Canada.

COMMISSION DE REFORME DU DROTT DU CANADA (1974) : Le Dedommagement et l'indemnisation : lamende, Documents de travail 5 et 6.

COMMISSION DE REFORME DU DROTT DU CANADA (1976) : La Participation communautaire da la réadaptation du délinquant, par Allen Linden, 245 p.

COMMISSION DE REFORME DU DROIT DU CANADA : Le dédommagement et l'indemnisation $>$ in Barreau 74,6 p.

CONSEIL DE L'EUROPE (1977) : Rapport final d'activité sur le dédommagement des victimes d'actes criminels, 88 p.

EDELHERTZ, H. et G. GEIS (1974) : Public Compensation to Victims of Crime, Etats-Unis, Praeger Publishers Inc., 309 p.

FALANDYSZ, L. (1979) : Wiktymologia, Varsovie, Biblioteka Wiedzy Wspolczesnej Omega, 193 p.

SOCIETÉ CANADIENNE DE CRIMINOLOGIE (1968) : \& La compensation aux victimes du crime et la restitution par les délinquants \$, 22 p.

WALLER, I. et N. OKIHIRO (1978) : Burglary : the Victim and the Public, Toronto, Les Presses de l'Université de Toronto, $190 \mathrm{p}$. 